\title{
Band Gap Characteristics of Planar Stretch-Dominated Thermal Expansion Lattice Metamaterial
}

\author{
HAibo YUE ${ }^{a}$, WeI WANG ${ }^{b, *}$, \\ WEIKAI XU ${ }^{b}$ AND SHASHA YUAN ${ }^{c}$ \\ ${ }^{a}$ Zhengzhou Institute of Mechanical and Electrical Engineering, \\ No. 126, Jingguang Middle Road, 450015, Zhengzhou City, Henan Province, China \\ ${ }^{b}$ School of Civil Engineering and Architecture, Suqian University, \\ No. 399, Huanghe South Road, 223800, Suqian City, Jiangsu Province, China \\ ${ }^{c}$ Shenyang Municipal Engineering Design Research Institute, \\ No. 37, Wencui Road, 110016, Shenyang City, Liaoning Province, China
}

Received: 31.08.2021 \& Accepted: 13.11.2021

Doi: $10.12693 /$ APhysPolA.140.438

*e-mail: starwei2002@163.com

\begin{abstract}
The current development of science and technology puts forward higher requirements for the versatility of materials. Based on the finite element method and Bloch theory, the band gap characteristics of the stretching-dominated lattice metamaterials are studied, the dispersion properties of elastic waves propagating in periodic lattice metamaterials are analyzed, and the effects of different configurations and material parameters on the band gap are discussed. Afterwards, an optimal design of the lattice metamaterial is proposed to maximize the relative band gap width. Results show that the thermal expansion configuration can also have a certain band gap characteristic while meeting the adjustable thermal expansion coefficient and certain rigidity requirements. Through reasonable material selection and shape design, it is expected to achieve dual goals of specific expansion properties and band gap design, so that composite material has better tunability and versatility.
\end{abstract}

topics: lattice metamaterial, coefficient of thermal expansion, band gap characteristics, optimal design

\section{Introduction}

Phononic crystal $(\mathrm{PnC})[1-3]$ is a kind of composite material with a spatial periodic structure. When acoustic/elastic waves pass through the PnCs, a band structure is formed, thus a passband or forbidden band will appear in a certain frequency range, in which the acoustic/elastic waves in the forbidden band frequency range cannot propagate. Due to the high potential of the PnCs band gap characteristics, they have broad application prospects in the fields of filtering, damping and noise reduction [4-9]. In general, generation of the band gap in PnCs is usually caused by Bragg scattering [10] or a local resonance mechanism [11]. According to these two mechanisms, the band structure can be controlled by changing material parameters such as density, modulus, acoustic impedance, and structure parameters such as lattice form and scatterer shape. Due to their great design ability, PnCs have become the subject of the research in various fields $[12,13]$.

The development of science and technology places even higher demands on the versatility of materials. For band gap materials, the tunability and multifunction of the band gap become the main issues considered. However, the main parameters affecting the band gap of PnCs are material parameters and structural parameters, which are difficult to change after the structure is determined. The current research direction is to change the properties and micro-structure of materials by applying external electric, magnetic, thermal and other physical fields, so as to realize the regulation of band gap performance [14]. On the other hand, the working environment of materials often has the coupling of light, electricity, magnetism, heat, force and other fields [15], which to meet the needs of further miniaturization and multifunction of phononic devices, so it puts forward new requirements for the properties of materials. For example, Phoxonic crystal (PXC) can achieve both the sound band gap and the optical band gap [16].

In aerospace and other fields, structures and materials often have to deal with the coupling effect of various physical fields in the service process, such as micromotor system, hypersonic protection system etc. As a result, they should not only cope with temperature changes, but also deal with the challenges related to vibration and 


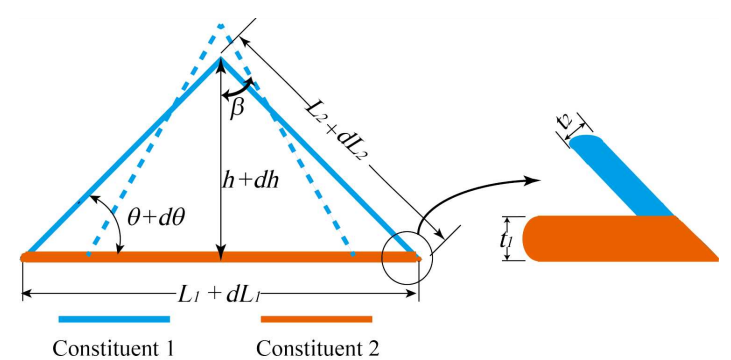

Fig. 1. Schematic of the primitive configuration. CTE is represented in the vertical direction.

Physical parameters of $\mathrm{Al}$ and invar [19]. TABLE I

\begin{tabular}{l|c|c|c|c}
\hline \hline Material & $\begin{array}{c}\text { Young } \\
\text { modulus } \\
{[\mathrm{GPa}]}\end{array}$ & $\begin{array}{c}\text { Density } \\
{\left[\mathrm{kg} / \mathrm{m}^{3}\right]}\end{array}$ & $\begin{array}{c}\text { Poisson } \\
\text { ratio }\end{array}$ & $\begin{array}{c}\mathrm{CTE} \\
{\left[\mathrm{ppm} /{ }^{\circ}\right]}\end{array}$ \\
\hline material 1 (Al) & 71 & 2810 & 0.33 & 23.0 \\
material 2 (invar) & 144 & 8100 & 0.25 & 1.1
\end{tabular}

noise. Thus, it is an important issue to consider the band gap characteristics when designing the structure with specific thermal expansion performance. Recently, the realization of specific coefficient of thermal expansion (CTE) materials has been widely covered [17-19]. For controlling the effective CTEs, the two most important configurations are curved- $[17,18,20,21]$ and stretchdominated $[19,22]$ lattice metamaterials, which depend on the axial tension-compression and bending deformation of the bar, respectively. Interestingly, both curved-dominated [23] and stretchdominated [24] thermal expansion materials are composed of lattice structures with different configurations that correspond to triangular, quadrilateral or hexagonal shapes. These topologies have special thermal expansion properties and may also have band gap characteristics. Therefore, it is a key problem to study the band gaps of thermal expansion unit cell with the desired CTEs.
In this paper, the energy band characteristics of a planar stretch-dominated lattice metamaterials are analyzed, as well as the band gap characteristics of single unit cell form with positive, negative and zero CTEs. Finally, an optimal design of the lattice metamaterial is proposed to maximize the relative band gap width (RBGW). The results show that the configuration of the unit cell has a great influence on structure. As the CTEs of the lattices is determined by the arrangement of different materials and base cells, a reasonable design of multifunctional lattice metamaterials can be realized.

\section{Models and methods}

\subsection{The dual-material unit cell}

In this paper, the dual-material lattice structure with respects to [19] is selected as the primitive configuration, as shown in Fig. 1. When planar lattices are heated, constituents 1 (hypotenuse members) and 2 (base member) deform in harmony with each other and then realize the special CTE in the vertical direction. According to the different arrangements, it can be divided into two configurations by shared vertex. In Category I, the vertices of the equilateral triangle share the point $\mathrm{M}$, and in Category II, the planar lattices share the base members and the vertex M points to the outside, as shown in Fig. 2.

According to [19], the constituent materials can be chosen as aluminum and invar, and their material parameters are listed in Table I. In general, when the temperature changes, the material parameters (such as modulus and density) also change which may have a given impact on their band gap characteristics.

However, the discussion in [23-26] shows that the influence of changing the properties of materials with temperature on the band gap is much smaller than that of deformation, especially when the temperature difference is not particularly large.
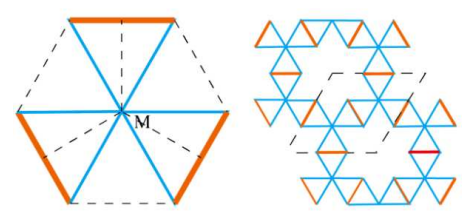

I-Triangular

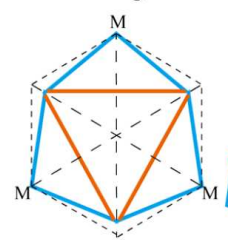

II-Triangular

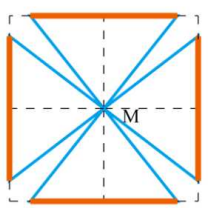

I-Square

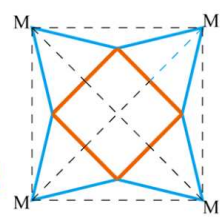

II-Square
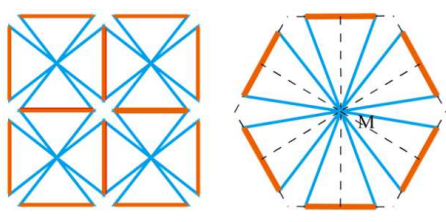

I-Hexagonal
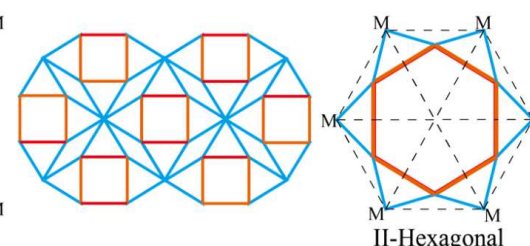
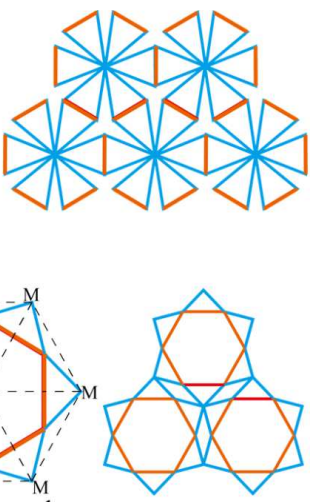

II-Hexagona

Fig. 2. Planar lattices in Category I and Category II for Trigonal, Tetragonal and Hexagonal cells. 


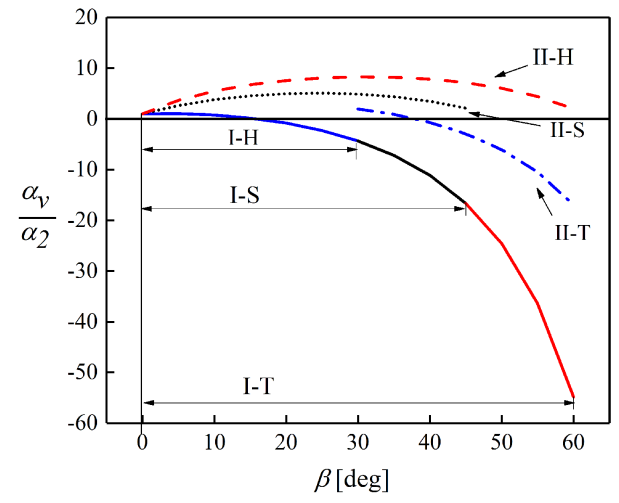

Fig. 3. Normalized effective CTE of the planar lattices with $\beta$.

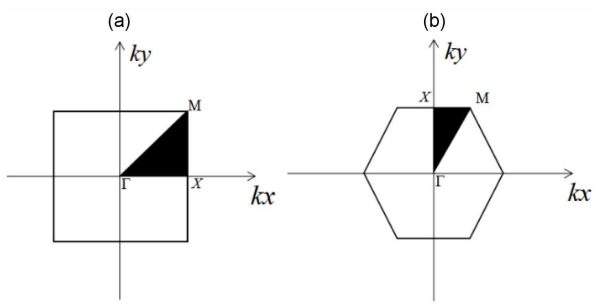

Fig. 4. First and irreducible Brillouin zone of (a) quadrilateral and (b) hexagonal protocells.

Thus, in this paper, we pay more attention to discuss the structural parameters and hope to achieve an optimal design. Now, I-T, I-S, I-H, and II-T, II-S, II-H are abbreviations for Triangular, Square, Hexagonal cells of Category I and II, respectively. Once the materials and structural dimensions are determined, the equivalent CTE of Category I and Category II can be expressed, respectively, as

$$
\frac{\alpha_{\nu}^{I}}{\alpha_{2}}=\frac{1-\frac{\alpha_{1}}{\alpha_{2}} \sin ^{2}(\beta)}{\cos ^{2}(\beta)}
$$

and

$$
\frac{\alpha_{v}^{I I}}{\alpha_{2}}= \begin{cases}\frac{1-\frac{1}{2} \frac{\alpha_{1}}{\alpha_{2}} \sin \left(2 \beta-\frac{\pi}{3}\right)\left(\frac{1}{\sqrt{3}}+\tan \left(\beta-\frac{\pi}{6}\right)\right)}{1-\frac{1}{2} \sin \left(2 \beta-\frac{\pi}{3}\right)\left(\frac{1}{\sqrt{3}}+\tan \left(\beta-\frac{\pi}{6}\right)\right)}, & \text { for II-T } \\ \frac{1+\frac{1}{2} \frac{\alpha_{1}}{\alpha_{2}} \cos (2 \beta)\left(1-\tan \left(\frac{\pi}{4}-\beta\right)\right)}{1+\frac{1}{2} \cos (2 \beta)\left(1-\tan \left(\frac{\pi}{4}-\beta\right)\right)}, & \text { for II-S } \\ \frac{1+\frac{1}{2} \frac{\alpha_{1}}{\alpha_{2}} \sin \left(\frac{2 \pi}{3}-2 \beta\right)\left(\sqrt{3}-\tan \left(\frac{\pi}{3}-\beta\right)\right)}{1+\frac{1}{2} \sin \left(\frac{2 \pi}{3}-2 \beta\right)\left(\sqrt{3}-\tan \left(\frac{\pi}{3}-\beta\right)\right)}, & \text { for II-H }\end{cases}
$$

where $\alpha_{1}$ and $\alpha_{2}$ are the CTEs of constituents 1 and 2 , respectively, and $\beta$ is the half of the apex angle representing the geometrical adjustable range of the configuration. The normalized effective CTE, i.e., $\alpha_{v} / \alpha_{2}$, can be drawn according to the change of $\beta$ as shown in Fig. 3. It can be seen that for different arrangement configuration one can achieve different CTEs and there may be different band gap characteristics. In particular, Category I lattices can be obtained by rotation of primitives. Therefore, according to (1), when $\beta$ is small, the lattice composed of the same primitives should have a unified CTE. However, when $\beta$ increases, the number of primitives will be constrained by their angles, such as no more than $360^{\circ}$. This means that I-Square and I-Hexagonal lattices will be limited by a geometric constraints of $\beta$. Namely, when $\beta$ is too large, these two configurations cannot be achieved. Regardless of this, when designing metamaterials with a specific CTE, there will be several different configurations to choose from, which take into account the band gap characteristic. Alternatively, once the configuration is determined, we can still change other parameters such as width of constituent material, to achieve better band gap design. Therefore, a discussion on the band gap characteristics of lattice configurations with different CTEs is required.

\subsection{Band gap analysis and calculation}

The equation governing the elastic wave in linear elasticity and isotropic passive medium is [27]

$$
\begin{aligned}
& \frac{1}{\rho(\boldsymbol{r})}\{\nabla \times[\mu(\boldsymbol{r}) \nabla \times \boldsymbol{u}(\boldsymbol{r})] \\
& \quad-\nabla[(\lambda(\boldsymbol{r})+2 \mu(\boldsymbol{r}))(\nabla \cdot \boldsymbol{u}(\boldsymbol{r}))]\}=\omega^{2} \boldsymbol{u}(\boldsymbol{r}),
\end{aligned}
$$

where $\omega$ is the angular frequency, $\boldsymbol{r}(x, y, z)$ denotes the position vector, $\boldsymbol{u}(\boldsymbol{r})$ is the displacement vector, $\nabla=(\partial / \partial x, \partial / \partial y, \partial / \partial z)$ is the gradient operator, $\lambda(\boldsymbol{r}), \mu(\boldsymbol{r})$ and $\rho(\boldsymbol{r})$ are the Lamé constants and material density, respectively, which have the same periodicity as the structure. According to the Bloch theorem, the displacement vector can be deduced as

$$
\begin{gathered}
\boldsymbol{u}(\boldsymbol{r}, t)=\mathrm{e}^{-\mathrm{i} \omega t} \sum_{\boldsymbol{G}} \boldsymbol{u}_{\boldsymbol{k}+\boldsymbol{G}} \mathrm{e}^{\mathrm{i}(\boldsymbol{k}+\boldsymbol{G}) \cdot \boldsymbol{r}}= \\
\mathrm{e}^{\mathrm{i}(\boldsymbol{k} \cdot \boldsymbol{r}-\omega t)} \boldsymbol{u}_{\boldsymbol{k}}(\boldsymbol{r}),
\end{gathered}
$$

where $\boldsymbol{k}=\left(k_{x}, k_{y}\right)$ is the wave vector of the first Brillouin zone and $\boldsymbol{G}$ represents the dimensionless space coordinate inverse lattice vector. Due to the translational periodicity, the spatial field distribution of one cell is the same as that of the entire lattice structure. According to crystallography, both the I-Square and II-Square protocells are quadrilateral lattices, and their irreducible Brillouin zones are the shadow shown in Fig. 4a. Otherwise, the protocells of I-Triangular, II-Triangular, I-Hexagonal, and II-Hexagonal lattices are hexagonal lattices, and their irreducible Brillouin zones are shown in Fig. 4b. For a band gap analysis, the dispersion curve of the lattice metamaterials can be obtained simply by traversing the boundary of the irreducible Brillouin zone. By using the commercial software COMSOL Multiphysics, the eigenvalue problem can be conveniently solved.

\section{Numerical simulation and discussion}

\subsection{Planar configurations selection with geometric constraints}

All planar configurations are obtained from the different triangular primitive arrangements shown in Fig. 1 and lead to different CTEs. Therefore, 

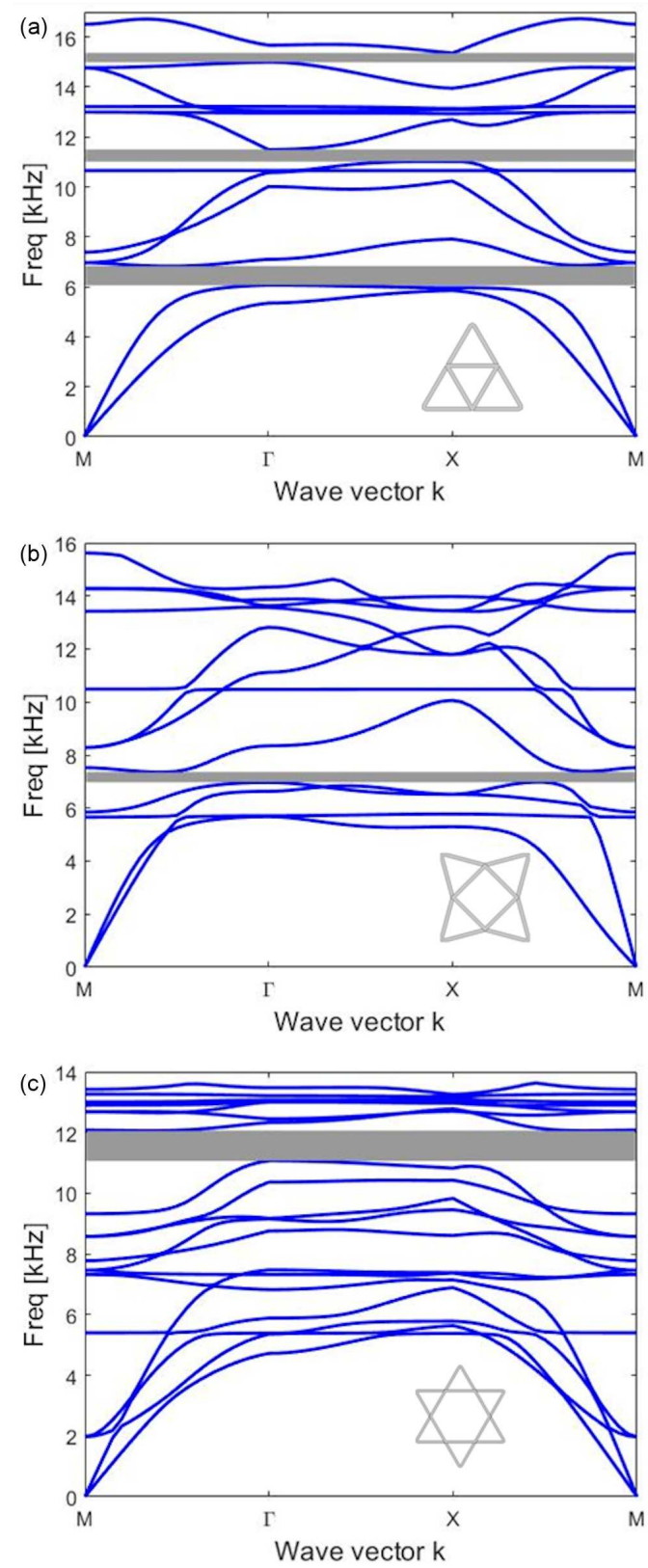

Fig. 5. Band gap diagrams of positive CTE models composed of (a) II-Triangular, (b) II-Square, and (c) II-Hexagonal.

the band gap characteristics of the lattice metamaterials should be discussed according to different CTEs. Some geometric constraints should also be considered, for example, when the angle $\beta$ is large, a hexagonal lattice is not possible.

\subsection{Band gap analysis of the metamaterials with positive CTE}

Firstly, the band gap of the configuration with positive CTE is studied. As can be seen in Fig. 3, the lattice configurations of Category I can only achieve a very small positive expansion at a small angle. Therefore, we selected three Category II configurations for the band gap analysis in the section. For example, while we select $\beta=30^{\circ}$, $L_{1}=39.3 \mathrm{~mm}, L_{2}=36.3 \mathrm{~mm}$, three lattice configuration of II-Triangular, II-Square and II-Hexagonal with the CTEs of 1.0, 4.08 and 7.64 are established. It can be seen that the effective CTEs varies with the different arrangement of the three Category II cells although using the same primitive configuration.

Figure 5 shows the bang gap diagrams of three lattice metamaterials. As observed in Fig. 5a, II-Triangular configuration has three complete band gaps at $6.05-6.9 \mathrm{kHz}, 11.0-11.5 \mathrm{kHz}$ and 15.0 $15.3 \mathrm{kHz}$, while II-Square and II-Hexagonal configuration have only one band gap at $6.98-7.35 \mathrm{kHz}$ and $11.07-12.05 \mathrm{kHz}$, respectively. All band gaps are not ideal, probably because the configuration of Category II is similar to truss structure with constituent 2 (invar) as the matrix and constituent 1 $(\mathrm{Al})$ as the inclusion, and the lower stiffness of the inclusion is not conducive to the formation of the band gap [27].

\subsection{Band gap analysis of the metamaterials with negative CTE}

According to (1) and (2) and Fig. 3, for lattice configurations of Category I, it is easier to obtain better negative expansion performance. In this section, we selected three Category I configurations for the band gap analysis. For example, selecting $\beta=26.5^{\circ}, L_{1}=36.2 \mathrm{~mm}, L_{2}=35.8 \mathrm{~mm}$, the three lattice configuration of I-Triangular, I-Square and I-Hexagonal are established. Unlike the previous section, both CTEs of these three configurations are -3.95 .

As can be seen in Fig. 6, I-Square configuration has almost no band gap, while I-Triangular configuration has one band gap at $7.0-7.8 \mathrm{kHz}$. In turn, I-Hexagonal configuration produces two band gaps at $6.4-6.9 \mathrm{kHz}$ and $8.0-10.9 \mathrm{kHz}$. Note that the I-Square configuration is similar to the traditional triangular lattice structure, which is not conducive to the generation of the band gap [28]. The I-Hexagonal lattice increases the number of connecting rods resulting in a wide band gap, but the I-Triangular appears to generate a band gap at a lower frequency.

\subsection{Band gap analysis of the metamaterials with zero CTE}

The configuration selection of the zero CTE is also determined by (1) and (2) and Fig. 3. In Fig. 3, it can still be seen that the Category I configurations are easier to obtain the zero expansion. Figure 7 shows the band gap diagrams of three zero-CTE models composed of I-Triangular, I-Square, and I-Hexagonal with $\beta=12.6^{\circ}$. Similar to Sect. 3.2, I-Square configuration has a very narrow ban gap, while multiple band gaps appear in both I-Triangular and I-Hexagonal structures. For example, I-Triangular has three 

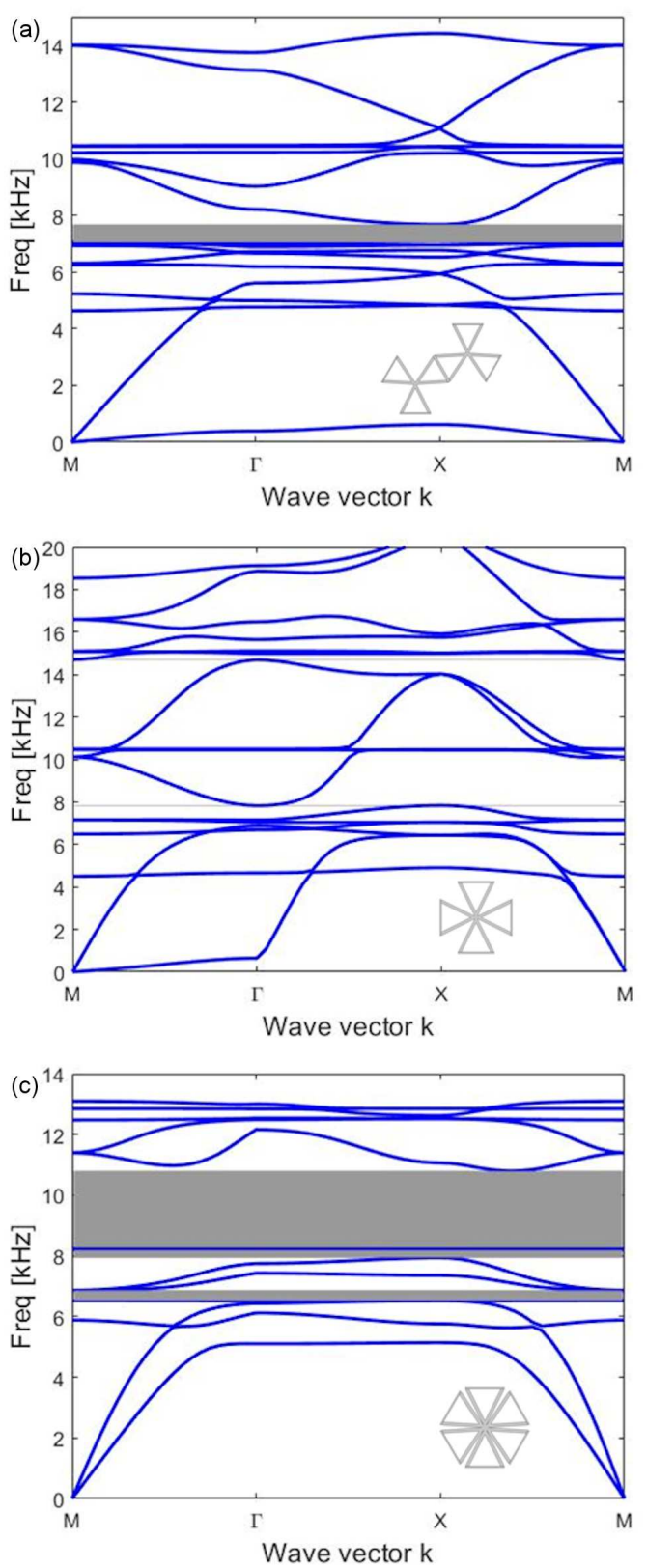

Fig. 6. Band gap diagrams of negative CTE models composed of (a) I-Triangular, (b) I-Square, and (c) I-Hexagonal.

band gaps at $5.71-6.17 \mathrm{kHz}, \quad 6.98-7.68 \mathrm{kHz}$ and $9.23-9.46 \mathrm{kHz}$; I-Hexagonal has also three band gaps at $6.43-6.58 \mathrm{kHz}, \quad 6.82-7.42 \mathrm{kHz}$ and $9.19-9.48 \mathrm{kHz}$, respectively. It is worth noting that the band gap of hexagonal cells becomes relatively narrow with the decrease of primitive angle, and the I-Triangular one still generates the band gap at a lower frequency.

\subsection{Optimal design of relative bandgap width (RBGW)}

All previous studies have shown that the geometrical parameters of the primitive configuration will lead to different band gap characteristics.
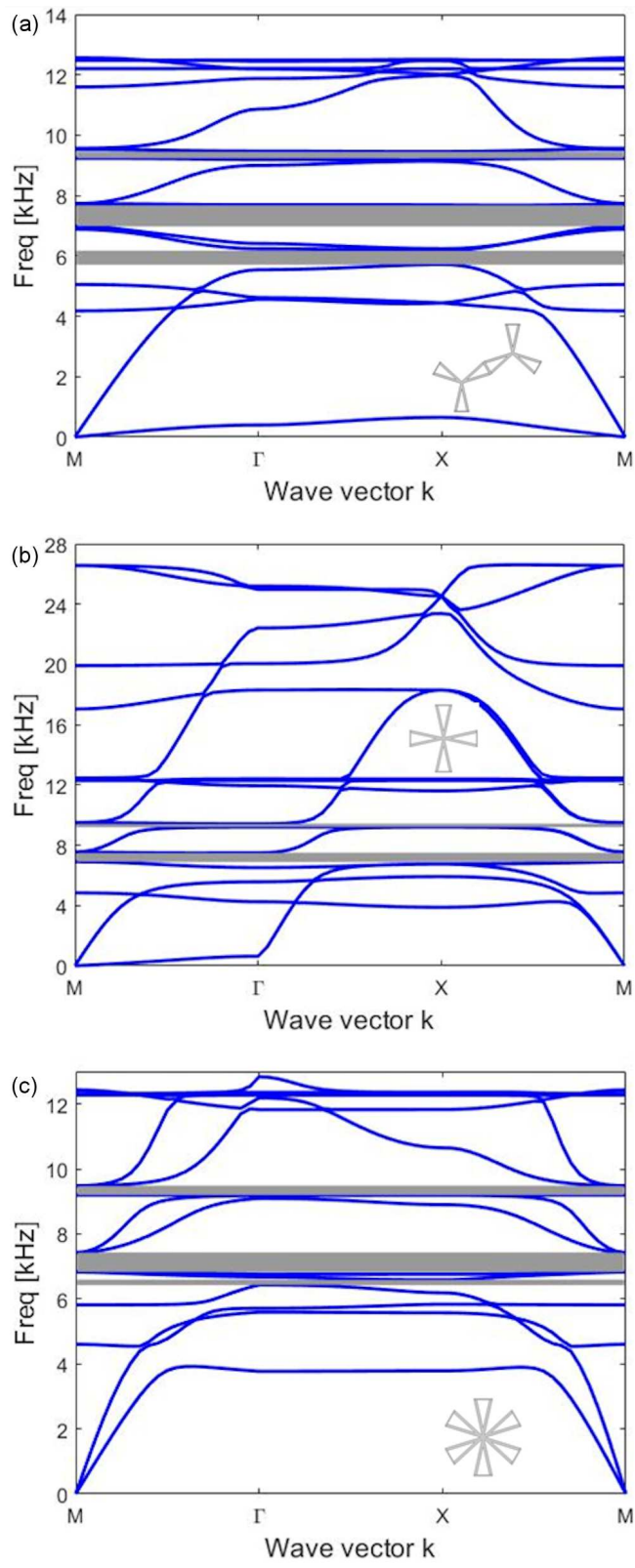

Fig. 7. Band gap diagrams of zero CTE models composed of (a) I-Triangular, (b) I-Square, and (c) I-Hexagonal.

Therefore, the optimal design of the band gap for this lattice metamaterials has become an interesting topic. In this section, we will present a case study of the band gap optimization design base on the following discussion.

Firstly, the relative band gap width (RBGW) is chosen $[29,30]$ as the target that means that the band gap of the metamaterial should have both a wider range and a lower frequency. The objective can be written as

$\operatorname{maximize} \quad F_{1}=2 \frac{\min \left(\omega_{n+1}(\boldsymbol{k})\right)-\max \left(\omega_{n}(\boldsymbol{k})\right)}{\min \left(\omega_{n+1}(\boldsymbol{k})\right)+\max \left(\omega_{n}(\boldsymbol{k})\right)}$, 

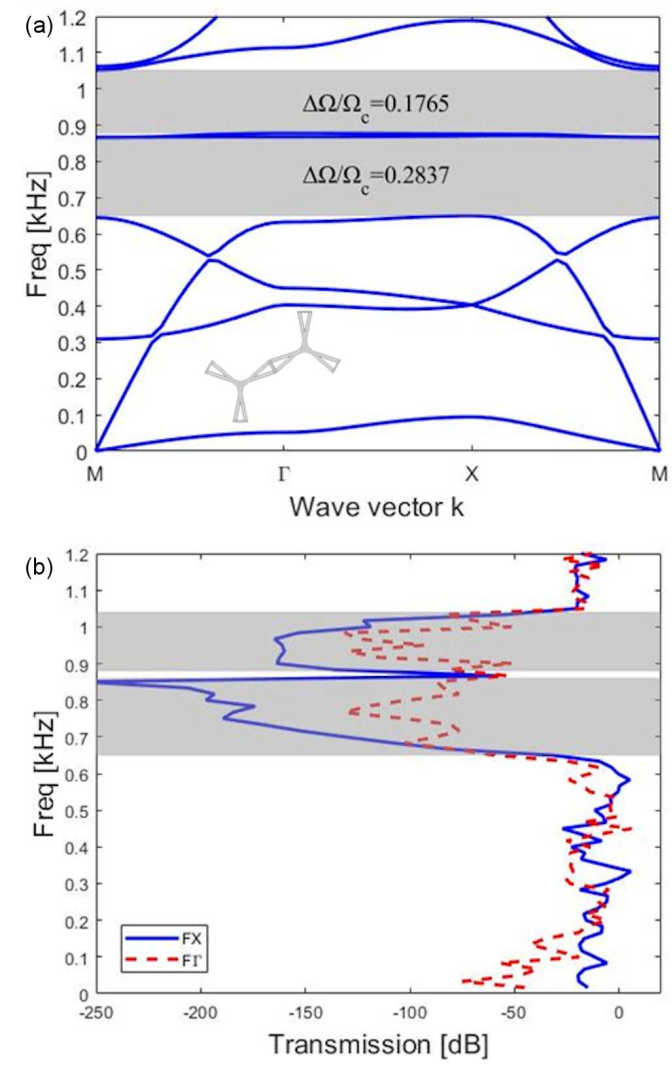

Fig. 8. (a) Band gap diagrams and (b) transmission spectra of the optimal result. Band gap is shaded in grey and the corresponding value is given.

where $\omega$ is the circular frequency, $n$ is the $n$-th band, and $\boldsymbol{k}$ denote the wave vector. As discussed above, for the sake of simplicity, we only select the I-Triangular configuration as the design prototype.

Secondly, it is well known that geometrical scaling without modifying the single-cell topological configuration will only change the frequency range but will not change the characteristics of the band gap. Therefore, we can only select a fixed cell size for optimal design. This means that when the angle $\beta$ is determined, the lengths of the two constituents, $L_{1}$ and $L_{2}$, are also determined.

Finally, in addition to the configuration of microstructure, the the distribution and amount of material also affect the band gap. The change of widths of the two constituent materials will affect the stiffness of the whole metamaterial and further affect the band gap characteristics [31]. In this case, taking $t_{1}$ and $t_{2}$, and the optimization problem can be formulated mathematically as

find $\boldsymbol{X}=\left(\beta, t_{1}, t_{2}\right)^{\mathrm{T}}$,

$\operatorname{maximize} F_{1}=2 \frac{\min \left(\omega_{n+1}(\boldsymbol{k})\right)-\max \left(\omega_{n}(\boldsymbol{k})\right)}{\min \left(\omega_{n+1}(\boldsymbol{k})\right)+\max \left(\omega_{n}(\boldsymbol{k})\right)}$,

s.t. $x_{\min } \leq x_{i} \leq x_{\max }, \quad i=1,2,3$.

Combining genetic algorithm (GA) and COMSOL, the optimization problem can be solved rapidly.
Figure 8 shows the band gap diagram and the transmission of the optimization result. The optimal configuration parameters are $\beta=11.26^{\circ}$, $t_{1}=5 \mathrm{~mm}$ and $t_{2}=0.5 \mathrm{~mm}$, with the effective CTE of 0.2108 . The maximum RBGW is 0.2837 , it is in the range of $0.65-0.87 \mathrm{kHz}$. Next, the second band gap is between $0.88-1.05 \mathrm{kHz}$, with the RBGW of 0.1765 . Compared with the above cases, it can be seen that the band gap is greatly reduced while ensuring a considerable bandwidth. Moreover, the results show that the transmission spectra are in good agreement with the band diagram.

\section{Conclusion}

The band gap characteristics of the planar stretch-dominated thermal expansion lattice composites are studied. Based on crystallography, different configurations of positive/negative/zero thermal expansion model are presented and their band gap characteristics are discussed. The results show that most of the configurations, including Category I and Category II, can produce a given band gap when exhibiting special CTEs. Geometrical parameters, such as the apex angle, widths of the constituent materials, exert a very significant impact on the band gap of the lattice metamaterials. For the stretch-dominated lattices in this paper, the positive expansion is easier to obtain by the Category II configurations, leading to weak band gap ability. Category I configuration can achieve a variety of negative expansion and zero expansion, and has a wider adjustment range. Based on this, an optimal design for geometric parameters is proposed at the end of the paper. The optimized configuration shows excellent band gap characteristics, which provides a theoretical basis for the design of metamaterials with dual objectives of thermal expansion/band gap.

Furthermore, this study can provide a benchmark for materials and equipment applied in many harsh environments, such as hypersonic thermal protection systems, communication satellites, and other spacecraft in the aerospace field. The temperature difference in these environments will exceed $200^{\circ} \mathrm{C}$, and its thermal expansion and band gap performance should be further in-depth research. On the other hand, a further full study on high-dimensional model should be carried out in future research, because the two-dimensional lattice metamaterials are difficult to be applied to the real structure. The influence of out-of-plane waves and their mixing with the in-plane waves for directions other than the high-symmetry ones should be discussed.

\section{Acknowledgments}

This work is supported by the National Natural Science Foundation of China (No. 11502149,11302135), Natural Science Foundation of Liaoning Province (Nos. 2019-ZD-0229, 2019-ZD0228 and 2019-ZD-0297), Scientific Research Fund 
of Liaoning Provincial Education Department (No. JYT19056), National Natural Science Foundation of Suqian City (No.K202124), Scientific Research Foundation of Suqian University. The financial contributions are gratefully acknowledged.

\section{References}

[1] M.S. Kushwaha, P. Halevi, L. Dobrzynski, Phys. Rev. Lett. 71, 2022 (1993).

[2] M.S. Kushwaha, Int. J. Mod. Phys. B 10, 977 (1996).

[3] J. Jensen, J. Sound Vibrat. 266, 1053 (2003).

[4] Y. Chen, F. Qian, L. Zuo, F. Scarpa, L. Wang, Extreme Mech. Lett. 17, 24 (2017).

[5] M.I. Hussein, M.J. Leamy, M. Ruzzene, Appl. Mech. Rev. 66, 040802 (2014).

[6] S.A. Cummer, J. Christensen, A. Alu, Nat. Rev. Mater. 1, 16001 (2016).

[7] G. Hu, L. Tang, A. Banerjee, R. Das, J. Vibrat. Acoust. 139, 011012 (2017).

[8] W.K. Xu, J.Y. Jing, M. Zhang, W. Wang, T.Z. Yang, Acta Mech. Solida Sin. 31, 775 (2018).

[9] Y.P. Tian, Z.H. Tan, X. Han, W.H. Li, J. Phys. D Appl. Phys. 52, 025102 (2019).

[10] H.G. Zhao, X.Y. Han, J.H. Wen, G. Wang, J. Mater. Sci. Eng. 21, 153 (2003).

[11] Z.Y. Liu, X.X. Zhang, Y.W. Mao et al., Science 289, 1734 (2000).

[12] Y. Huang, S.T. Liu, J. Zhao, Struct. Multidiscip O. 48, 487 (2013).

[13] W.K. Xu, J.Y. Ning, Z.B. Lin et al., Mater. Today Commun. 22, 100801 (2020).

[14] X.S. Wen, J.H. Wen, D.L. Yu, Phononic Crystals National Defence Industry Press, Beijing (China) 2009.
[15] T. Vasileiadis, J. Varghese, V. Babacic et al., J. Appl. Phys. 129, 160901 (2021).

[16] H.W. Dong, Y.S. Wang, C.Z. Zhang, IEEE Photonics J. 9, 1 (2017).

[17] J. Lehman, R. Lakes, J. Intel. Mater. Syst. Str. 23, 1263 (2012).

[18] J. Lehman, R. Lakes, Compos. Struct. 107, 654 (2014).

[19] K. Wei, H. Chen, Y. Pei et al., J. Mech. Phys. Solids 86, 173 (2016).

[20] J. Lehman, R. Lakes, Int. J. Mech. Mater. Des. 9, 213 (2013).

[21] Y.C. Zhang, Y.J. Liang, S.T. Liu et al., Acta Mech. Solida Sin. 35, 507 (2019).

[22] K. Wei, Y.M. Pei, (in Chinese) Chin. Sci. Bull. 62, 47 (2017).

[23] W.K. Xu, S.C. Lv, C.L. Liu et al., J. Appl. Phys. 130, 085106 (2021).

[24] S.C. Lv, W.K. Xu, L. Bai et al., Appl. Phys. A 127, 425 (2021).

[25] K. Bertoldi, M.C. Boyce, Phys. Rev. B 77 , 439 (2008).

[26] K. Bertoldi, M.C. Boyce, Phys. Rev. B 78, 2599 (2008)

[27] S.T. Liu, X.F. Cao, D. He, W.Z. Su, Chin. J. Appl. Mech. 24, 213 (2007).

[28] B. Wu, R.J. Wei, H.Y. Zhao et al., Acta Mech. Solida Sin. 23, 255 (2010).

[29] O. Sigmund, J.S. Jensen, Philos. Trans. RSoc. Lond. A 361, 1001 (2003).

[30] H.W. Dong, X.X. Su, Y.S. Wang, C.Z. Zhang, Struct. Multidisc. Optim. 50, 593 (2014).

[31] C.A. Steeves, S. Lucato, H. Ming et al., J. Mech. Phys. Solids 55, 1803 (2007). 